\title{
基于非线性薛定谔方程的时间序列去噪
}

\author{
雷一凡 ${ }^{1)}$, 宋刚1)，高成勇 ${ }^{2}$, 包芳勋 ${ }^{1 *}$, 张云峰 3$)$ \\ 1) (山东大学数学学院 济南 250100) \\ 2) (山东大学物理学院 济南 250100) \\ 3) (山东财经大学计算机科学与技术学院 济南 250014) \\ (fxbao@sdu.edu.cn)
}

\begin{abstract}
摘 要: 针对噪声污染严重的时间序列, 提出一种基于动态随机共振效应的去噪方法. 通过具体分析非线性光学系统 中的动态随机共振效应，推导出非线性薛定谔方程中的非线性扰动项，得到用于描述非线性系统中的信号传播模型， 并应用于时间序列去噪. 首先将归一化的时间序列信号输入模型系统，然后通过自适应粒子群优化算法确定系统传播 方程中的各项参数, 最后利用分步傅里叶方法求解传播方程的数值解, 作为系统的输出. 对于一些典型的时间序列信 号, 与现有的去噪方法相比, 文中方法在信噪比上平均提高 $0.3 \sim 3.7 \mathrm{~dB}$, 在均方根误差上平均降低 $0.03 \sim 0.11$, 该方法在 时间序列去噪上具有更好的效果.
\end{abstract}

关键词: 非线性薛定谔方程; 动态随机共振; 自适应粒子群优化; 时间序列去噪

中图法分类号: TP391.41ＤOI: 10.3724/SP.J.1089.2021.18671

\section{Time Series Denoising Using Nonlinear Schrödinger Equation}

\author{
Lei Yifan ${ }^{1)}$, Song Gang ${ }^{1)}$, Gao Chengyong ${ }^{2)}$, Bao Fangxun ${ }^{1)^{*}}$, and Zhang Yunfeng ${ }^{3)}$ \\ 1) (School of Mathematics, Shandong University, Jinan 250100) \\ 2) (School of Physics, Shandong University, Jinan 250100) \\ 3) (School of Computer Science and Technology, Shandong University of Finance and Economics, Jinan 250014)
}

\begin{abstract}
In order to remove noise in time series with quantities of noise, a denoising method based on dynamical stochastic resonance is presented. By analyzing the dynamical stochastic resonance in nonlinear optical systems, the nonlinear disturbance in nonlinear Schrödinger equation is derived, the propagation model is obtained and applied to time series denoising. Firstly, the time series signal is normalized as system input. Then the parameters of the system propagation equation are determined by the adaptive particle swarm optimization algorithm. The numerical solution of the propagation equation is obtained by the split step Fourier method as system output. Compared with the existing denoising methods, proposed method improves signal-to-noise ratio by $0.3-3.7 \mathrm{~dB}$, reduces by $0.03-0.11$ on average for typical time series signals. Experimental results show the better denoising ability of proposed method.
\end{abstract}

Key words: nonlinear Schrödinger equation; dynamical stochastic resonance; adaptive particle swarm optimization; time series denoising

收稿日期: 2020-09-18; 修回日期: 2020-11-16. 基金项目：国家自然科学基金(61672018，61972227,61873117, U1609218); 山东 省自然科学基金(ZR201808160102, ZR2019MF051); 山东省重点研发计划(GG201710090122, 2017GGX10109, 2018GGX101013); 山东 省高等学校优势科研人才团队培育计划基金. 雷一凡(1996-), 男, 硕士研究生, 主要研究方向为图像处理; 宋刚(1993一), 男, 博士 研究生, 主要研究方向为 CAGD、图像处理; 高成勇(1971一), 男, 博士, 副教授, 主要研究方向为光学测试计量技术及仪器、非线性 光学; 包芳勋(1968一), 男, 博士, 教授, 博士生导师, CCF 会员, 论文通讯作者, 主要研究方向为函数逼近、分形、CAGD; 张云峰 (1977一), 男, 博士, 教授, 博士生导师, CCF 会员, 主要研究方向为 CAGD、图像处理. 
随着社会学、工程学、生物医学、系统科学等 领域中复杂数据的迅速增长, 时间序列分析在数 据分析领域越来越被重视. 而当数据过于嘈杂时, 准确分析时间序列数据将极其困难. 因此, 时间序 列去噪成为一个很重要的问题, 并被大量应用于 公共交通 ${ }^{[1]}$ 、卫星定位 ${ }^{[2]}$ 和金融评估 ${ }^{[3]}$ 等领域.

在时间序列去噪的研究中，小波分解是一种常 用的方法. 基于小波的理论，诸如自适应小波 ${ }^{[4]}$ 、经 验模态分解 ${ }^{[5]}$ (empirical mode decomposition, EMD) 和集合经验模态分解 ${ }^{[6]}$ (complete ensemble EMD, CEEMD)等改进方法相继提出. 这些方法的思想大 致可以归纳如下：(1) 分解, 将信号以某种规律进 行分解; (2) 去噪, 将分解得到的各个信号的分量分 别进行去噪处理; (3) 重构, 将处理完的各个分量以 该规律逆变换，重新组合得到去噪后的信号.

小波去噪中常用方法为小波阈值去噪．首先 通过选择适当的小波基函数以及分解层数，对含 噪信号进行小波变换分解；然后选取合适的阈值 对分解得到的小波系数进行硬阈值或软阈值处理; 最后将信号重构, 从而达到去噪的效果. Alt 等 ${ }^{[7]}$ 结 合机器学习，提出了一种自适应小波阈值去噪方 法, 通过参数化函数的训练结果推断出小波收缩 函数, 从而降低信号中噪声的标准差.

EMD 和 CEEMD 等方法采用相关系数准则确 定分界本征模态函数(intrinsic mode function, IMF), 从而将原始信号分解为多个 IMF 分量, 利用包含 真实信号的 IMF 分量重构信号, 达到去噪的目的. $\mathrm{Li}$ 等 $^{[8]}$ 结合 CEEMD 以及主成分分析法，将信号分 解为多个 IMF 分量, 对于同时包含有真实信号以 及噪声信号的 IMF 分量进行主成分分析, 将处理 后的包含真实信号的 IMF 分量进行重构, 达到去 噪的目的.

不同于小波分解的思想，稀疏优化考虑信号 的特性, 提供了另外一种从含有噪声的信号中分 离出真实信号的方法. 这种方法认为, 在能够保留 绝大部分信息的前提下，信号经过某些变换后是 稀疏的，即可以由有限个非零采样点来重构信号， 而噪声往往是不可稀疏表示的. 因此, 在构造特定 正则化约束条件后，信号的去噪问题等价于在被 噪声污染的信号中寻找到最优的稀疏表示, 使目 标约束达到最优. 基于这种思想, Selesnick 等 ${ }^{[9]}$ 结 合低通滤波和稀疏优化方法，将信号分解为低频 分量和高频分量, 在噪声能量聚集的高频分量中, 进一步利用稀疏优化方法提取出真实信号的信息, 利用这些信息和在真实信号能量聚集的低频分量
进行重构, 从而得到去噪信号.

不同于以上分解重构的思想，随机共振是一 种直接将噪声能量转化为信号能量的方法, 与小 波去噪的思想相比, 由于没有对信号进行任何形 式的滤波操作, 在理想状态下的非线性系统中, 利 用随机共振的现象进行去噪不会损失信号的能量.

随机共振现象最早由 Benzi 等 ${ }^{[10]}$ 在研究古冰 川周期性问题时发现，当信号在某些非线性系统 中传输时, 信号中的噪声会与信号之间发生某种 非线性耦合，导致噪声与信号之间发生能量交换， 当噪声的强度达到一定量时, 信号与噪声之间的 能量转换将达到最高, 从而使系统输出信号信噪 比(signal to noise ratio, SNR)达到最大. Benzi 等将 这种非线性耦合的现象命名为随机共振.

随机共振是普遍存在于非线性系统中的一种 动力学行为, 关于随机共振的理论与应用也得到 了大量的研究, 单稳态系统 ${ }^{[11]}$ 、多稳态系统 ${ }^{[12]}$ 和阈 值系统 ${ }^{[13]}$ 等模型相继提出, 它们在信号处理 ${ }^{[14]}$ 、量 子物理 ${ }^{[15]}$ 、光学 ${ }^{[16]}$ 和通信 ${ }^{[17]}$ 等多个领域都有重要 的应用.

在传统随机共振系统中，信号与系统间的耦 合是固定的, 由初始信号与势阱所确定. 这种固定 的耦合意味着当信号中存在的噪声比较复杂时, 噪声的能量不能完全转换为信号的能量. 而在非 线性光学中, 光束在非线性介质中的传输一方面 会改变介质的性质, 另一方面由于非线性介质性 质的改变, 系统的势会发生改变, 从而改变光束之 间的耦合. 这是一种动态的随机共振过程. 基于上 述理论, Dylov 等 ${ }^{[18]}$ 利用光折变非线性晶体构建了 一套光学系统, 用于观测非线性光学中的随机共 振现象. 在该系统中, 非线性耦合使噪声的能量能 够转换为信号的能量, 从而恢复被噪声所淹没的 图像信号。

鉴于以上观察，本文具体分析上述过程中光 束的传播行为, 利用非线性薛定谔方程描述信号 在系统中的传播过程, 并利用这一过程中发生的 随机共振效应调整参数, 使非线性耦合达到最佳, 从而达到信号去噪的效果.

在参数的调整上，选择常用于参数寻优问题 的粒子群优化 (particle swarm optimization, PSO)算 法调整去噪算法的参数. 粒子群优化算法是一种 模拟鸟群受食行为的演化计算方法, 由于其算法 具有效率高且易于实现的特点, 在目标分类 ${ }^{[19]}$ 及 特征选择 ${ }^{[20]}$ 等方面有着广泛的应用.

在解决实际问题时，经典 PSO 算法容易陷人 
局部最优, 并且当模型较为复杂时, 优化效率不够 高，算法的收玫速度较慢. 为了避免局部最优，同 时提高优化效率，本文提出基于自适应学习策略 的粒子群优化(PSO with adaptive learning strategy, PSO-ALS)算法, 对参数进行优化, 与经典的 PSO 算法相比，它有着更快的收玫速度和更高的效率.

根据本文算法，将时间序列数据作为系统的 输人，在选取特定的非线性项以及系统参数后，其 在时间序列去噪上能达到很好的效果.

\section{1 相关理论与方法}

薛定谔方程是一种用于描述微观粒子在势力 场内行为的方程. 在非线性光学系统中, 考虑光伏 效应对系统空间电荷场的影响, 系统的势力场也 是变化的，此时用于描述系统中光束传播行为的 方程为非线性薛定谔方程. 在确定具体传播方程 后, 利用分步傅里叶方法求解方程的数值解. 在模 型的进一步优化上，利用 PSO-ALS 算法调节传播 方程的参数项，使去噪效果达到最佳.

\section{1 非线性薛定谔方程}

设 $\phi(x, t)$ 为微观粒子的运动状态, 称为微观粒 子的波函数，则对于给定的势力场 $V(x)$, 有薛定 谔方程 $\mathrm{i} \hbar \frac{\partial \phi(x, t)}{\partial t}=-\frac{\hbar^{2}}{2 m} \Delta \phi(x, t)-V(x) \phi(x, t)$ 成立.

当势力场的变化依赖波函数时, 有非线性薛定 谔方程 $\mathrm{i} \phi_{t}+\Delta \phi+f\left(\phi, \nabla_{\phi}\right)=0$ 成立. 其中, $f\left(\phi, \nabla_{\phi}\right)$ 表示非线性作用项，其与势力场在波函数的影响下 的变化有关.

在光学系统中, 光的传播速度与频率 $\omega$ 有关. 考虑光的色散现象, 并忽略高阶项的影响, 光信号 在介质中的传播方程为

$$
\mathrm{i} \frac{\partial \phi}{\partial t}-\frac{k^{\prime \prime}}{2} \frac{\partial^{2} \phi}{\partial x^{2}}=0 .
$$

其中, $k^{\prime \prime}=\frac{\partial^{2} k}{\partial \omega^{2}}, k=\frac{n \omega}{c} ; n$ 表示介质的折射率.

考虑 Kerr 非线性作用, 介质的折射率随着空 间电荷场的变化而改变, 此时 $n=n_{0}+n_{\mathrm{e}}|\phi|^{2}, n_{0}$ 和 $n_{\mathrm{e}}$ 分别表示平行和垂直于电场方向的折射率. 上 述方程即为

$$
\mathrm{i} \frac{\partial \phi}{\partial t}-\frac{k^{\prime \prime}}{2} \frac{\partial^{2} \phi}{\partial x^{2}}+g|\phi|^{2} \phi=0
$$

其中, $g=\frac{\pi n_{\mathrm{e}}}{\lambda \varepsilon^{2}}$ 表示非线性系数; $\lambda$ 表示光在介质
中的波长; $\varepsilon=\Delta \omega / \omega$.

对式(1)进行归一化, 并忽略高阶扰动项和能 量的损耗，得到标准非线性薛定谔方程

$$
\mathrm{i} \frac{\partial \phi}{\partial \xi}+\frac{1}{2} \frac{\partial^{2} \phi}{\partial s^{2}}+|\phi|^{2} \phi=0 .
$$

\section{2 薛定谔方程的数值解}

薛定谔方程的数值解可以由分步傅里叶方法 得出, 对于一般形式的薛定谔方程, 有

$$
\mathrm{i} \frac{\partial \phi}{\partial t}=-\frac{1}{2} \frac{\partial^{2} \phi}{\partial x^{2}}+V(x) \phi, \phi(x, 0)=\phi_{0}(x)
$$

考虑在很短的时间间隔 $[\tau, \tau+\Delta t]$ 内，假设此 时 $\phi(x, \tau)$ 已知, 且 $-\frac{1}{2} \frac{\partial^{2} \phi}{\partial x^{2}}$ 可以忽略, 即 $\phi_{x x} \approx 0$, 式 (2)即为

$$
\mathrm{i} \frac{\partial \phi}{\partial t}=V(x) \phi
$$

对于时间 $t=\tau+\Delta t$, 式(3)有如下形式的解.

$$
\phi(x, \tau+\Delta t)=\mathrm{e}^{(-\mathrm{i} \Delta t V(x)) \phi(x, \tau)}
$$

接下来，重新考虑式(2)并忽略 $V(x)$ 的影响, 此时式(2)即为

$$
\mathrm{i} \frac{\partial \phi}{\partial t}=-\frac{1}{2} \frac{\partial^{2} \phi}{\partial x^{2}}
$$

在时间间隔 $[\tau, \tau+\Delta t]$ 内, 对式(5)进行傅里叶 变换, 有 $\frac{\partial \hat{\phi}}{\partial t}=\mathrm{i} \mathcal{F}\left(\frac{\partial^{2} \phi}{\partial x^{2}}\right)=-i k^{2} \hat{\phi}$.

傅里叶变换和傅里叶逆变换的定义为

$$
\left\{\begin{array}{l}
\hat{h}(k)=\mathcal{F}(h)=\int_{-\infty}^{\infty} h(x) \mathrm{e}^{-2 \pi \mathrm{i} k x} \mathrm{~d} x \\
h(k)=\mathcal{F}(\hat{h})=\int_{-\infty}^{\infty} \hat{h}(k) \mathrm{e}^{2 \pi \mathrm{i} k x} \mathrm{~d} k
\end{array} .\right.
$$

此时, $\hat{\phi}$ 的解析解为

$$
\hat{\phi}(k, \tau+\Delta t)=\mathrm{e}^{-i k^{2} \Delta t} \hat{\phi}(k, \tau)
$$

对式(6)进行傅里叶逆变换, 得到

$$
\phi(k, \tau+\Delta t)=\mathcal{F}^{-1}\left(\mathrm{e}^{-\mathrm{i} k^{2} \Delta t} \hat{\phi}(k, \tau+\Delta t)\right)
$$

结合式(4)和式(7), 对于薛定谔方程式(2), 它 的解有递推关系

$$
\phi(k, \tau+\Delta t)=\mathcal{F}^{-1}\left(\mathrm{e}^{-\mathrm{i} k^{2} \Delta t} \mathcal{F}\left(\mathrm{e}^{-\mathrm{i} \Delta t V(x)} \phi(x, \tau)\right)\right) .
$$

在初值条件 $\phi(x, 0)=\phi_{0}(x)$ 下，可以得到每个 时间步长的数值解.

\subsection{PSO 算法}

PSO 算法主要源于对鸟群社会行为的研究与 仿真. 鸟群在受食时, 最简单有效的方法就是搜索 
离食物最近的鸟所在的区域，通过个体间的协助 和信息共享实现群体进化. 即在受食过程中, 总有 某只鸟对食物所在地有较好的侦察力, 能获得较 为准确的食物位置信息. 在此信息的指引下，其他 同伴会逐渐向该位置聚集，最终寻找到食源.

PSO 算法是将群体中的个体看做多维搜索空 间中的一个粒子，每个粒子代表优化问题的一个 可能解，其特征信息用位置、速度和适应度 3 种指 标描述, 适应度由适应度函数计算得到, 其值的大 小表示粒子的优劣. 粒子以一定的速度运动, 根据 自身及其他粒子的移动经验，即个体历史最优位 置和种群历史最优位置, 改变移动的方向和距离, 不断迭代寻找较优区域，从而完成在全局搜索空 间中的寻优过程. PSO 算法的数学描述如下.

在 $D$ 维搜索空间中, 每个粒子 $i(i=1,2, \cdots, N)$ 与速度 $V_{i}=\left[v_{i}^{1}, v_{i}^{2}, \cdots, v_{i}^{D}\right]$ 和位置 $X_{i}=\left[x_{i}^{1}, x_{i}^{2}, \cdots, x_{i}^{D}\right]$ 有关. 起初, 粒子以随机的速度在搜索空间上初始 化. 在搜索过程中, 每个粒子 $i$ 在第 $D$ 维上的 $V_{i}$ 和 $X_{i}$ 按照

$$
\left\{\begin{array}{l}
v_{i}^{d}=v_{i}^{d}+c_{1} r_{1}^{d}\left(p_{i}^{d}-x_{i}^{d}\right)+c_{2} r_{2}^{d}\left(g^{d}-x_{i}^{d}\right) \\
x_{i}^{d}=x_{i}^{d}+v_{i}^{d}
\end{array}\right.
$$

更新. 其中, $d=1,2, \cdots, D$; 正常数 $c_{1}$ 和 $c_{2}$ 表示加 速度系数; $r_{1}^{d}$ 和 $r_{2}^{d}$ 分别表示在 $[0,1]$ 服从均匀分布 的 2 个随机数; $p_{i}$ 表示粒子 $i$ 的个体历史最优位 置; $g$ 表示种群历史最优位置, 即迄今为止所有粒 子发现的全局最优位置. 根据式(8)可知粒子的速 度由 3 个部分构成：(1) 粒子对上一次演化速度的 继承，反映了粒子运动的惯性; (2) 粒子的“自我认 知”部分, 由个体历史最优位置 $p_{i}$ 引导更新, 表示 粒子先前的飞行经验对随后飞行方向的影响; (3) “社会认知”部分, 由种群历史最优位置 $g$ 引导更 新, 代表整个种群先前的飞行经验对每个粒子随 后飞行方向的影响.

\section{2 时间序列去噪算法}

在 Dylov 等 ${ }^{[18]}$ 构建的光学系统中, 非线性耦合 发生在光折变晶体 $\mathrm{SBN}: 60(\mathrm{Sr} 0.6 \mathrm{Ba} 0.4 \mathrm{Nb} 2 \mathrm{O} 6)$ 中. 系统非线性反映在晶体折射率 $\delta_{n}=k E\langle I\rangle /(1+\langle I\rangle)$ 的改变上. 其中， $E$ 表示空间电荷场； $I$ 表示光束 的功率密度; $k=r_{33}\left(1+\left\langle I_{0}\right\rangle\right)$ 为常数, 由晶体的电 光系数 $r_{33}$ 与空间均匀人射光强度 $\left\langle I_{0}\right\rangle$ 决定. 输人
的光信号为带有分辨率板信息的相干光.

在上述光学系统中, Dylov 等 ${ }^{[18]}$ 通过调节外加 电场的强度来改变空间电荷场的分布，从而调节 系统的非线性系数. 随着非线性系数的增大, 系统 输出的图像变得越来越清晰, 意味着 SNR 逐渐提 高, 当到达一个峰值后, 继续增大非线性系数, 输 出图像的质量开始下降. 这是典型的随机共振现 象的特征.

接下来利用非线性薛定谔方程具体分析该系 统中信号的传播方程, 并利用基于自适应学习策 略的 PSO 算法对方程选取适当的参数.

\section{1 非线性系统中信号的传播方程}

在上述系统中，设晶体中光束沿 $\mathrm{z}$ 方向传播, 沿 $x$ 方向偏振, 考虑一束固定光束的传播, 该光束 在传播路径上的折射率变化表示为

$$
\left(n_{\mathrm{e}}^{\prime}\right)^{2}=n_{\mathrm{e}}^{2}-n_{\mathrm{e}}^{2} r_{33} E_{\mathrm{sc}} .
$$

其中, $n_{\mathrm{e}}^{\prime}$ 表示无外界扰动下的晶体折射率; $r_{33}$ 表 示电光系数; $E_{\mathrm{sc}}=E_{\mathrm{sc}} \hat{x}$ 表示晶体中的空间电荷场. 光束中电场分量满足 Helmholtz 方程，即

$$
\nabla^{2} \boldsymbol{E}+\left(k_{0} n_{\mathrm{e}}^{\prime}\right)^{2} \boldsymbol{E}=0
$$

其中, $k_{0}=2 \pi / \lambda_{0}, \lambda_{0}$ 表示真空中光的波长. 电场 强度 $\boldsymbol{E}=\hat{x} \phi(x, z) \exp (\mathrm{i} k z)$, 此时式(9)表示为傍轴 近似方程 $\mathrm{i} \phi_{z}+\frac{1}{2 k} \phi_{x x}-\frac{\sigma k_{0}}{2}\left(n_{\mathrm{e}}^{3} r_{33} E_{\mathrm{sc}}\right) \phi=0$. 其中, $k=k_{0} n_{\mathrm{e}}$

归一化坐标 $\xi=z / z_{0}=z / k x_{0}^{2}, s=x / x_{0}$, 得到

$$
\mathrm{i} \frac{\partial \phi}{\partial \xi}+\frac{1}{2} \frac{\partial^{2} \phi}{\partial s^{2}}-\alpha \phi=0
$$

其中, $\alpha=\frac{1}{2} \sigma k_{0}^{2} x_{0}^{2} n_{\mathrm{e}}^{4} r_{33} E_{\mathrm{sc}}, E_{\mathrm{sc}}$ 表示晶体中的空间 电荷场. 在考虑外加电场对空间电荷场分布的影 响下, 有

$$
\mathrm{i} \frac{\partial \phi}{\partial \xi}+\frac{1}{2} \frac{\partial^{2} \phi}{\partial s^{2}}-\gamma \frac{|\phi|^{2}}{1+|\phi|^{2}} \phi=0
$$

其中, $\gamma=\frac{1}{2} \sigma k_{0}^{2} x_{0}^{2} n_{\mathrm{e}}^{4} r_{33} E_{0} ; E_{0}$ 表示在外加电场作 用下的空间电荷场.

\section{2 参数的选取}

式(11)描述的是非线性系统中信号的传播过 程. 首先, 为了调节系统中非线性耦合的强度, 需 要调节势力场的参数 $\gamma$; 其次, 由于势力场强度的 变化是动态的, 信号的传播不会趋于稳态, 需要确 定信号在何时达到 SNR 峰值. 因此, 为了得到具 
体的数值解, 需要确定的参数有非线性项系数 $\gamma$ 与传播距离 $\xi$, 前者决定非线性耦合的强度, 后者 决定初始信号在非线性系统中的迭代次数.

图 1 所示为改变不同参数的情况下输出信号 的 SNR 变化. 可以看到, 随着非线性项系数的改 变, 信号的 SNR 会出现先增大后减小的趋势, 这 正是随机共振的特性. 而随着传播时间的改变, 输 出信号的 SNR 在某一时刻达到峰值, 这是非线性 介质中光束传播先聚焦后散焦造成的. 在具体算法 中, 本文构造了 PSO-ALS 算法确定最优参数.

不同于经典 PSO 算法中直接将搜索空间中的 粒子作为一个群体, 首先通过快速搜索聚类的方
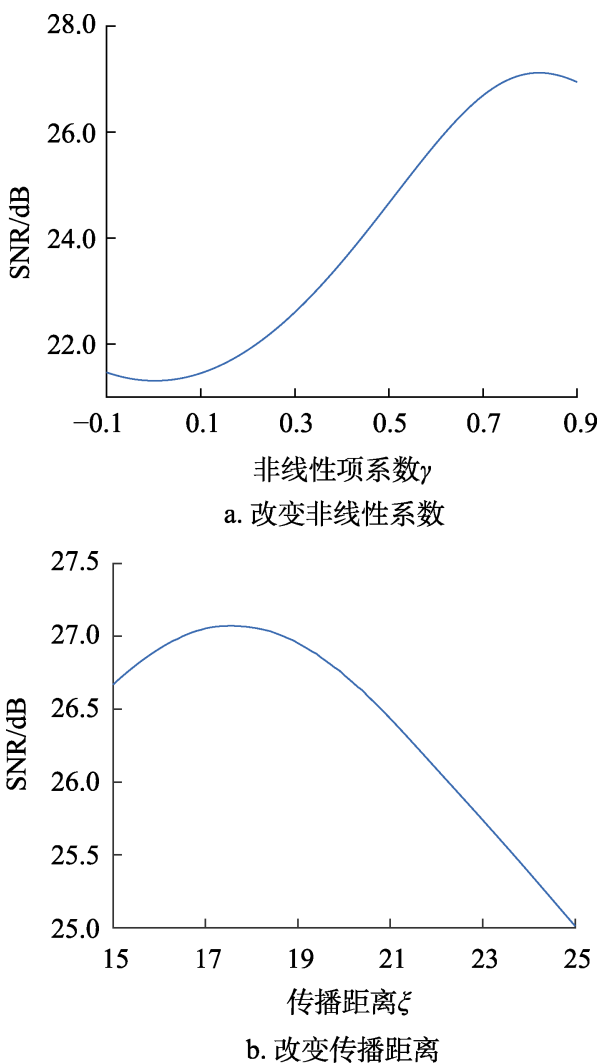

图 1 改变不同参数输出信号的 SNR 变化
法，自适应地将整个种群划分为若干个子群，相应 地，每个子群中的粒子可分为普通粒子和局部最 优粒子. 然后，对于普通粒子，使用局部最优粒子 引导其社会学习部分, 以增强种群多样性; 对于局 部最优粒子, 使用所有子群的局部最优粒子的平 均信息引导其社会学习部分, 通过信息交换进一 步促进种群多样性.

普通粒子主要负责在其子群所在的区域内进 行开发，其学习策略定义为

$$
x_{i}^{d}=\omega x_{i}^{d}+c_{1} r_{1}^{d}\left(p_{i}^{d}-x_{i}^{d}\right)+c_{2} r_{2}^{d}\left(g_{c}^{d}-x_{i}^{d}\right)
$$

其中， $\omega$ 表示惯性权重； $c_{1}$ 和 $c_{2}$ 表示加速度系数; $r_{1}$ 和 $r_{2}$ 表示在 $[0,1]$ 区间均匀分布的 2 个随机数; $g_{c}$ 表示子群 $c$ 中的局部最优粒子. 不同于传统 PSO 算法的学习策略，用局部最优粒子代替全局最优 粒子指导普通粒子的更新, 如此, 每个子群能够学 习到更多不同的搜索信息，以增加种群多样性.

局部最优粒子一方面用于引导普通粒子的学 习, 另一方面负责勘探其他潜在的更优的搜索区 域, 其学习策略为

$$
x_{i}^{d}=\omega x_{i}^{d}+c_{1} r_{1}^{d}\left(p_{i}^{d}-x_{i}^{d}\right)+c_{2} r_{2}^{d}\left(\frac{1}{C} \sum_{c=1}^{C} g_{c}^{d}-x_{i}^{d}\right)
$$

其中, $C$ 为子群的个数.

可以看到，式(13)中社会学习部分中的 $g_{c}$ 被 $C$ 个 $g_{c}$ 的平均信息所代替, 考虑局部最优粒子是 最有可能找到最优解的粒子, 它们的平均信息可 以整合关于最优解的有价值的信息. 利用这些优 越的信息指导粒子的更新, 可以加快收玫速度. 图 2 所示为该算法的示意图.

将上述算法用于优化式(11)中的参数: 非线性 项系数 $\gamma$ 与传播距离 $\xi$, 选择 SNR 作为目标函数, 得到使 SNR 达到最大的 $\gamma$ 和 $\xi$ 参数, 用于去噪算 法中. 算法流程如下.

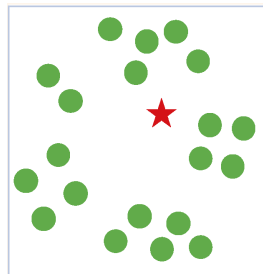

初始化

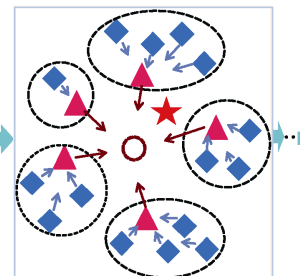

第1次演化

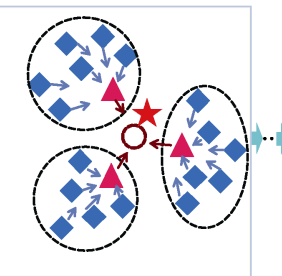

第 $i$ 次演化

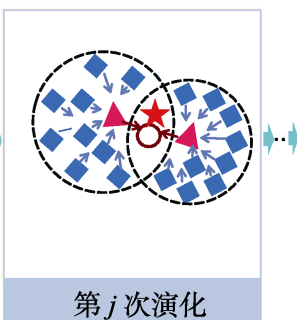

第 $j$ 次演化

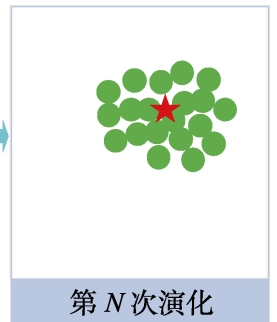

第 $N$ 次演化

太全局最优解位置; 0 局部最优解的平均位置;

- 普通粒子位置; $\Delta$ 局部最优粒子位置; 原始粒子位置

图 2 基于自适应学习策略的粒子群优化算法示意图 
输人. 待优化参数 $\gamma, \xi$.

输出. 优化后的参数 $\gamma, \xi$.

Step1. 初始化位置, 得到 $\gamma$ 和 $\xi$ 的初始值.

Step2. 聚类算法划分子群，得到 $C$ 个子群以及每 个子群中的局部最优粒子.

Step3. 对于每个种群中的普通粒子，按式(12)所示 的学习策略更新, 局部最优粒子按式(13)所示的学习策 略更新

Step4. 评价每个子群中的粒子, 更新局部最优粒 子和局部最优的参数值.

Step5．根据局部最优粒子的信息，更新 $\gamma$ 和 $\xi$.

Step6. 重复 Step1 Step5, 直到达到终止条件.

\section{3 实验与分析}

结合上述传播方程，采用分步傅里叶方法求 解非线性薛定谔方程的数值解，利用基于 PSOALS 算法确定最优参数. 算法流程如下.

输人. 含噪声时间序列信号 $s(i)$.

输出. 去噪信号 $\hat{s}(i)$.

Step1. 归一化输人信号, 进行傅里叶变换, 得到频 率域信号。

Step2. 利用 PSO-ALS 算法得到最优参数 $\gamma$ 和 $\xi$.

Step3. 利用分步傅里叶方法求数值解.

Step4. 进行傅里叶逆变换, 得到输出信号.

为了直观地反映去噪效果，利用模拟信号对 本文算法进行验证，模拟信号表示为

$$
y=2 \sin \left(\frac{2 \pi t}{800}\right) \times \sin \left(\frac{2 \pi t}{250}\right)+2 \sin \left(\frac{2 \pi t}{600}\right)+\sin \left(\frac{2 \pi t}{50}\right) .
$$

添加的噪声为高斯白噪声, $\mathrm{SNR}=4.0 \mathrm{~dB}$.

图 3 给出了本文方法对于上述模拟信号的去 噪效果. 如图 3c 所示, 原始信号被强度为 $4.0 \mathrm{~dB}$ 的加性高斯白噪声完全淹没，在经过去噪处理之 后，得到的信号波形与原始信号的波形相吻合. 可以看到，在强度较高的高斯白噪声下，该方法 对于非周期正弦信号的去噪效果比较理想, 系统 中发生的能量转移过程将高频的噪声信号完全转 移到了纯净信号中. 需要注意的是, 由于忽略了 扩散效应中信号能量的损失，信号的能量在非线 性系统中传播时没有损耗，当传播过程中没有引 人额外噪声时, 输人信号与输出信号的能量保持 恒定.

图 4 对比了利用传统 PSO 算法优化参数的去 噪结果和利用PSO-ALS 算法优化参数的去噪结果, 本次实验中, 2 种优化算法的迭代次数均固定为 50 .
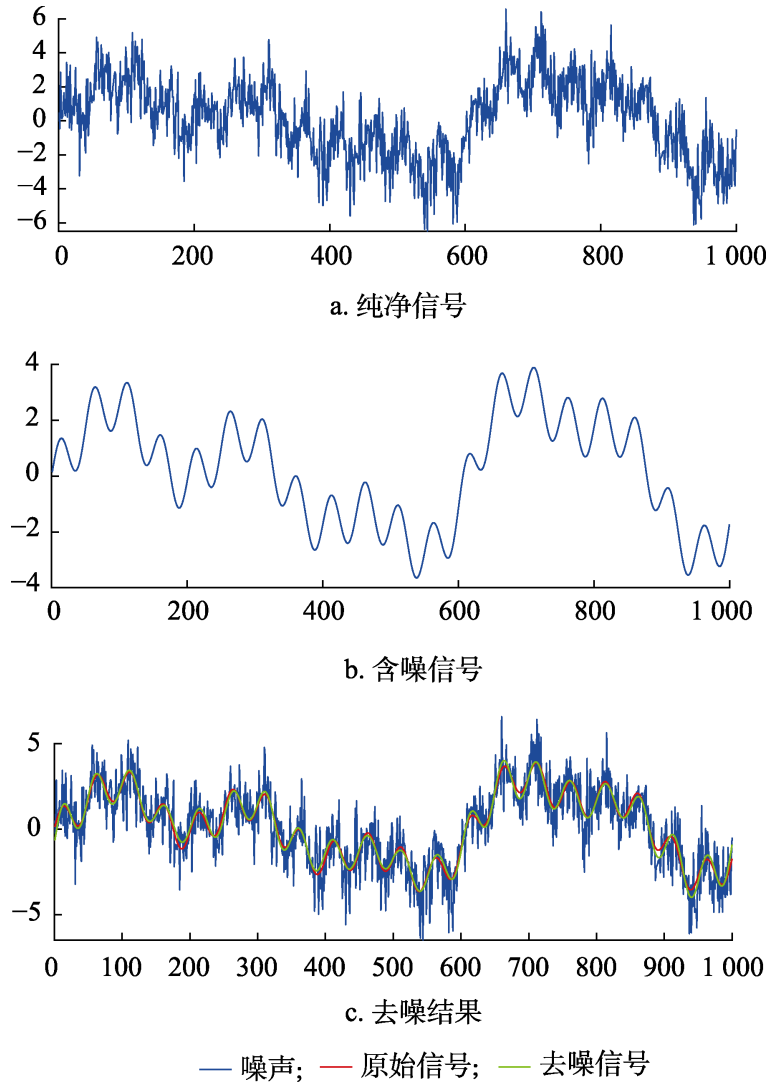

图 3 模拟信号去噪效果

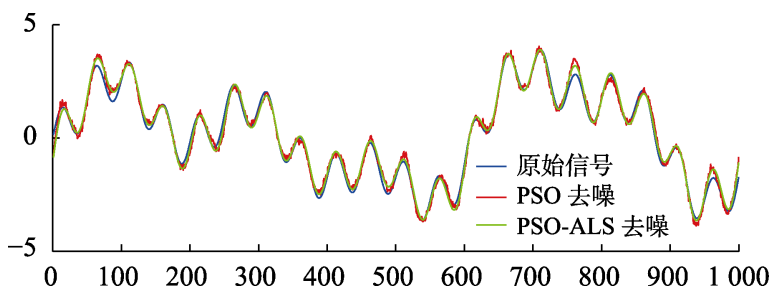

图 4 模拟信号去噪效果对比

可以看到，利用传统 PSO 算法的去噪信号中仍然 存在高频噪声的扰动, 而利用 PSO-ALS 算法得到 的去噪信号则更为平滑, 说明 PSO-ALS 算法比传 统 PSO 算法在参数的优化上效果更好.

接下来，利用文献[21]方法生成 Blocks 信号和 Heavisine 信号, 分别使用小波软阈值方法、基于 CEEMD 的方法 ${ }^{[8]}$ 、基于稀疏优化的方法 ${ }^{[9]}$ 以及本 文方法对以上信号进行去噪. 如图 5 所示, 第 1 行 为 Blocks 信号 (信号 1 ), 第 2 行为 Heavisine 信号(信 号 2), 第 3 行(信号 3)和第 4 行(信号 4)中的原始信 号分别由 Blocks 信号与 Heavisine 信号求和以及作 差产生. 纯净信号中引人的噪声为高斯白噪声.

为了直观地反映各方法的去噪效果, 选择均 方根误差(root mean square error, RMSE)以及 SNR 作为评价指标. 其中, RMSE 用于衡量去噪结果与 
纯净信号之间的偏差，计算方法为

$$
\operatorname{RMSE}=\sqrt{\frac{1}{2 N} \sum_{i=1}^{N}(s(i)-\hat{s}(i))^{2}} .
$$

其中, $N$ 为样本数据个数; $s(i)$ 为输人的纯净信 号; $\hat{s}(i)$ 为输出的去噪信号. RMSE 越小, 去噪结 果越接近于原始信号.

SNR 用于计算去噪结果中仍然存在的噪声的 强度, 计算方法为 $\mathrm{SNR}=10 \lg \frac{S}{N} \mathrm{~dB}$. 其中, $S$ 表示 不含噪声的纯净信号的功率; $N$ 表示噪声功率, $N=S-\hat{S} ; \hat{S}$ 表示得到的去噪信号.

基于 CEEMD 的方法 ${ }^{[8]}$ 是改进基于小波的方 法，为了公平起见，取小波方法与基于 CEEMD 的 方法 ${ }^{[8]}$ 中的阈值均为 $\lambda=\sigma \sqrt{2 \ln N}$. 这是一种在小 波软阈值去噪中常用的阈值取值, 且能达到比较 好的效果. 本文方法中的参数由粒子群算法确定.
以上几种方法的去噪结果如图 5 所示, 每种方 法对于每个信号的去噪结果的 RMSE 和 SNR 如表 1 所示. 可以看到，小波软阈值去噪方法在非周期 信号以及强噪声的情况下, 去噪效果较差, 输出的 去噪信号不够平滑, 评价指标也均不如其他方法. 在处理类似于 Blocks 信号这种具有稀疏特性的信 号时，基于稀疏优化的方法 ${ }^{[9]}$ 能够得到更加理想的 效果，与其他方法相比，该方法能够得到更为平滑 的去噪信号, 但同时也造成了部分信号的损失, 因 此在 SNR 上该方法的效果较差. 而文献[8]方法与 本文方法效果比较接近, 均能达到平滑曲线的效 果, 同时能够更好地保持信号的能量, 在 RMSE 和 SNR 的指标上, 2 种方法的结果也较为接近, 本文 方法在大部分情况下要略优于文献[8]方法，仅在 最后一种信号去噪的结果上, 文献[8]方法略优于 本文方法.
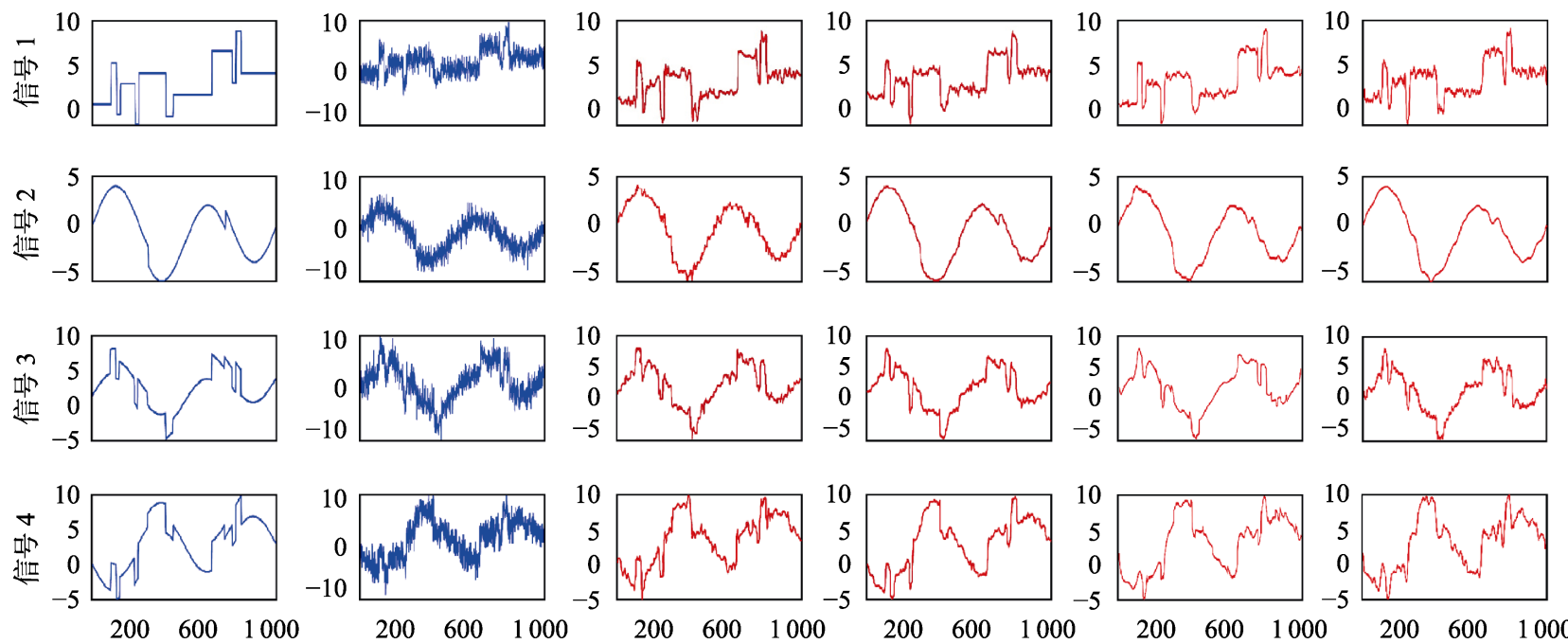

a. 原始信号

b. 含噪信号

c. 小波 ${ }^{[7]}$

d. 文献[8]

e. 文献[9]

f. 本文

图 5 不同方法去噪效果对比

表 1 不同方法去噪结果的评价指标对比

\begin{tabular}{|c|c|c|c|c|c|c|c|c|}
\hline \multirow{2}{*}{ 信号 } & \multicolumn{2}{|c|}{ 小波 ${ }^{[7]}$} & \multicolumn{2}{|c|}{ 文献[8] } & \multicolumn{2}{|c|}{ 文献[9] } & \multicolumn{2}{|c|}{ 本文 } \\
\hline & RMSE & $\mathrm{SNR} / \mathrm{dB}$ & RMSE & $\mathrm{SNR} / \mathrm{dB}$ & RMSE & $\mathrm{SNR} / \mathrm{dB}$ & RMSE & $\mathrm{SNR} / \mathrm{dB}$ \\
\hline 信号 1 & 0.50 & 11.9 & 0.47 & 16.1 & 0.38 & 15.9 & 0.42 & 16.8 \\
\hline 信号 2 & 0.35 & 18.6 & 0.25 & 20.8 & 0.30 & 19.9 & 0.21 & 23.1 \\
\hline 信号 3 & 0.47 & 16.6 & 0.54 & 16.9 & 0.51 & 16.9 & 0.44 & 17.5 \\
\hline 信号 4 & 0.72 & 14.4 & 0.45 & 20.7 & 0.59 & 18.2 & 0.53 & 19.1 \\
\hline 平均值 & 0.51 & 15.4 & 0.43 & 18.6 & 0.45 & 17.7 & 0.40 & 19.1 \\
\hline
\end{tabular}

注. 表中粗体字为最优评价指标结果.

\section{4 结 语}

根据非线性光学中的动态随机共振效应, 本
文分析并给出了描述信号在非线性光学系统中传 播的非线性薛定谔方程, 利用该方程, 提出了一种 用于时间序列去噪的方法. 该方法比其他随机共 
振方法去噪效果更好. 与现有的时间序列去噪方 法相比, 该方法能够取得比较理想的去噪效果, 对 于薛定谔方程在时间序列上的应用具有启发性.

\section{参考文献(References):}

[1] Boto-Giralda D, Díaz-Pernas F J, González-Ortega D, et al. Wavelet-based denoising for traffic volume time series forecasting with self-organizing neural networks $[\mathrm{J}]$. ComputerAided Civil and Infrastructure Engineering, 2010, 25: 530-545

[2] Montillet J P, Tregoning P, McClusky S, et al. Extracting white noise statistics in GPS coordinate time series[J]. IEEE Geoscience and Remote Sensing Letters, 2013, 10(3): 563-567

[3] Alrumaih R M, Al-Fawzan M A. Time series forecasting using wavelet denoising an application to saudi stock index[J]. Journal of King Saud University-Engineering Sciences, 2002, 14(2): 221-234

[4] Zhang W, Wang X, Ge L L, et al. Noise reduction in ECG signal based on adaptive wavelet transform[C] //Proceedings of the IEEE Engineering in Medicine and Biology 27th Annual Conference. Los Alamitos: IEEE Computer Society Press, 2005: 2699-2702

[5] Huang N E, Shen Z, Long S R, et al. The empirical mode decomposition and Hilbert spectrum for nonlinear and nonstationary time series analysis $[\mathrm{J}]$. Proceedings of The Royal Society A Mathematical Physical and Engineering Sciences, 1998, 454: 903-995

[6] Li J, Liu C, Zeng Z F, et al. GPR signal denoising and target extraction with the CEEMD method[J]. IEEE Geoscience and Remote Sensing Letters, 2015, 12(8): 1615-1619

[7] Alt T, Weickert J. Learning a generic adaptive wavelet shrinkage function for denoising[OL]. [2020-09-18]. https://arxiv. org/abs/1910.09234

[8] Li Y Y, Xu C J, Yi L, et al. A data-driven approach for denoising GNSS position time series[J]. Journal of Geodesy, 2018, 92: 905-922

[9] Selesnick I W, Graber H L, Pfeil D S, et al. Simultaneous low-pass filtering and total variation denoising[J]. IEEE Transactions on Signal Processing, 2014, 62(5): 1109-1123
[10] Benzi R, Sutera A, Vulpiani A. The mechanism of stochastic resonance[J]. Journal of Physics A: Mathematical and General, 1981, 14: 453-457

[11] Kang Y M, Xu J X, Jin W Y. Stochastic synchronization and aperiodic stochastic resonance of a unidirectionally coupled single-mode optical system[J]. International Journal of Nonlinear Sciences and Numerical Simulation, 2005, 6(1): 19-24

[12] Evstigneev M, Reimann P, Pankov V, et al. Stochastic resonance in monostable overdamped systems[J]. Europhysics Letters, 2004, 65(1): 7-12

[13] Mitaim S, Kosko B. Evidence for stochastic resonance in threshold systems based on mutual information[C] //Proceedings of the IEEE International Conference on Industrial Technology. Los Alamitos: IEEE Computer Society Press, 2002: 1315-1320

[14] Duan F B, Xu B H. Parameter-induced stochastic resonance and baseband binary PAM signals transmission over an AWGN channel[J]. International Journal of Bifurcation and Chaos, 2003, 13(2): 411-425

[15] Grifoni M, Hänggi P. Nonlinear quantum stochastic resonance[J]. Physical Review E, 1996, 54(2): 1390-1401

[16] Monifi F, Zhang J, Özdemir Ş K, et al. Optomechanically induced stochastic resonance and chaos transfer between optical fields[J]. Nature Photonics, 2016, 10: 399-405

[17] Chapeau-Blondeau F, Godivier X. Stochastic resonance in nonlinear transmission of spike signals: an exact model and an application to the neuron[J]. International Journal of Bifurcation and Chaos, 1996, 6(11): 2069-2076

[18] Dylov D V, Fleischer J W. Nonlinear self-filtering of noisy images via dynamical stochastic resonance[J]. Nature Photonics, 2010, 4(5): 323-328

[19] Tran B, Xue B, Zhang M J. Variable-length particle swarm optimization for feature selection on high-dimensional classification[J]. IEEE Transactions on Evolutionary Computation, 2019, 23(3): 473-487

[20] Tan T Y, Zhang L, Neoh S N, et al. Intelligent skin cancer detection using enhanced particle swarm optimization[J]. Knowledge-Based Systems, 2018, 158: 118-135

[21] Donoho D L, Johnstone I M. Ideal spatial adaptation by wavelet shrinkage[J]. Biometrika, 1994, 81(3): 425-455 